2016-08

\title{
Changes in the EEG amplitude as a biomarker for early detection of Alzheimer's disease
}

\author{
Al-Nuaimi, Ali H. Husseen
}

http://hdl.handle.net/10026.1/13251

\subsection{9/embc.2016.7590869}

2016 38th Annual International Conference of the IEEE Engineering in Medicine and Biology Society (EMBC)

IEEE

All content in PEARL is protected by copyright law. Author manuscripts are made available in accordance with publisher policies. Please cite only the published version using the details provided on the item record or document. In the absence of an open licence (e.g. Creative Commons), permissions for further reuse of content should be sought from the publisher or author. 


\title{
Changes in the EEG Amplitude as a Biomarker for Early Detection of Alzheimer's Disease
}

\author{
Ali H. Al-nuaimi, Student Member, IEEE, Emmanuel Jammeh, Lingfen Sun and Emmanuel Ifeachor
}

\begin{abstract}
The rapid increase in the number of older people with Alzheimer's disease (AD) and other forms of dementia represents one of the major challenges to the health and social care systems. Early detection of AD makes it possible for patients to access appropriate services and to benefit from new treatments and therapies, as and when they become available. The onset of AD starts many years before the clinical symptoms become clear. A biomarker that can measure the brain changes in this period would be useful for early diagnosis of AD. Potentially, the electroencephalogram (EEG) can play a valuable role in early detection of $\mathrm{AD}$. Damage in the brain due to $\mathrm{AD}$ leads to changes in the information processing activity of the brain and the EEG which can be quantified as a biomarker. The objective of the study reported in this paper is to develop robust EEG-based biomarkers for detecting AD in its early stages. We present a new approach to quantify the slowing of the EEG, one of the most consistent features at different stages of dementia, based on changes in the EEG amplitudes $\left(\triangle \mathrm{EEG}_{\mathrm{A}}\right)$. The new approach has sensitivity and specificity values of $100 \%$ and $88.88 \%$, respectively, and outperformed the Lempel-Ziv Complexity (LZC) approach in discriminating between $\mathrm{AD}$ and normal subjects.
\end{abstract}

Keywords: Alzheimer's disease, dementia, EEG biomarkers, early diagnosis.

\section{INTRODUCTION}

$\mathrm{AD}$ is a progressive, neurodegenerative disorder that affects cognitive brain functions [1]. The rapid increase in the number of people living with $\mathrm{AD}$ and other forms of dementia represents a significant challenge to our health and social care systems and to society. Currently, there are over 46.8 million individuals with dementia worldwide at an annual cost of US $\$ 818$ billion, and this is projected to reach 74.7 million by 2030 with an annual cost of US\$ 2 trillion [2].

Early detection of AD is important to enable patients and their families to have proper access to available health and social care [3]. It also makes it possible for patients to gain maximum benefits from new treatments and therapies, as and when they become available, to mitigate against disease progression before irreversible damage is caused to brain cells [4].

Brain changes caused by AD are believed to start 10 to 20 years before the clinical symptoms are observed [4]. There is a need for a reliable, low-cost, easy to use tool for early detection of $\mathrm{AD}$. This requires a biomarker that detects brain changes due to $\mathrm{AD}$ in this period. Biomarkers, such as those derived from computerized tomography (CT) and magnetic resonance imaging (MRI) are useful for $\mathrm{AD}$ diagnosis, but neuroimaging is expensive, is available only in specialist centres, and it may not be suitable for certain patients (e.g. patients with pacemakers or certain implants [5]).

Potentially, the EEG can play a valuable role in the early detection of AD. Damage to nerve cells/pathways in the brain due to $\mathrm{AD}$ causes changes in the information processing activity of the brain and the EEG and this can be quantified as a biomarker [6][7]. Changes in the information processing activity of the brain are thought to be reflected in the information content of the EEG [6][7]. In AD patients, the EEG is characterized by variations in the complexity measures, mean frequency, and in the coherences between cortical regions [8]. EEG has a high temporal resolution and provides valuable information about brain dynamics in $\mathrm{AD}$ [9]. Many techniques exist for deriving AD biomarkers from the EEG [10]. However, time domain-based approaches are potentially one of the most reliable ways to derive robust EEG biomarkers for AD [1][6][11].

The slowing of the EEG is one of the most consistent features at different stages of dementia [11][12][13] and the extent of the slowing may be quantified as a biomarker of AD. In this study, we present a new approach to quantify the slowing of the EEG in the time domain by measuring changes in the EEG amplitudes. The changes in the amplitudes over time may be viewed as the mean velocity of the EEG [14]. The approach is easy to implement and is computationally efficient.

We used the new method to discriminate between AD and normal subjects and obtained a sensitivity and specificity values of $100 \%$ and $88.88 \%$, respectively. We compared the performance of the new approach to the LZC method. LZC is a nonparametric, non-linear measure of complexity for finite length sequences [15]. The LZC approach produces a good biomarker for $\mathrm{AD}$ detection [16] and is used to analyse brain function, brain information transmission, and EEG complexity in patients with AD [17]. The new approach outperformed the LZC approach using the same datasets.

The paper is arranged as follows. In Section II, the methodology used in the study is described. In Section III, the materials (including the datasets and EEG recordings) are described. Section IV presents the results and Section V concludes the paper.

\section{METHODOLOGY}

In our approach, changes in the amplitudes are used as a measure of the slowing of the EEG. In particular, the sum of the differences between adjacent amplitudes of EEG values per second [14] is determined from:

$$
\Delta \mathrm{EEG}_{\mathrm{A}}=\frac{\sum \Delta x}{\Delta t}
$$


where $\Delta \mathrm{x}$ represents the difference between adjacent amplitudes of the EEG in one second and $\Delta t$ denotes the time interval:

$$
\begin{gathered}
\Delta x=x_{i+1}-x_{i} \\
\Delta t=t_{i+1}-t_{i}
\end{gathered}
$$

where $x_{i}$ and $x_{i+1}$ are the current and next EEG amplitude values, respectively, and $t_{i}$ and $t_{i+1}$ represent the corresponding times $i$.

$\triangle \mathrm{EEG}_{\mathrm{A}}$ is first computed using Equation "(1)" for each EEG channel. The mean $\triangle \mathrm{EEG}_{\mathrm{A}}$ for the channel is then computed as,

$$
M_{C}=\left(\sum_{i=1}^{N} \Delta E E G_{A}\right) / N
$$

where $M_{C}$ is the mean value of $\triangle \mathrm{EEG}_{\mathrm{A}}$, and $N$ is the number of samples for the EEG signal.

The process of deriving the biomarker is divided into two phases - a development phase and a testing phase. In the development phase, two reference feature vectors are created from the mean $M_{C}$ values for all the EEG channels (one for normal and the other for $\mathrm{AD}$ groups). In the testing phase, one feature vector is created for each new subject.

The Euclidean distance measure is then used to discriminate between $\mathrm{AD}$ and normal subjects in the classification stage, as,

$$
D_{i}=\sqrt{\left(V-V_{i}\right)^{2}}
$$

where $D_{i}$ is the distance between the reference feature vector $(V)$ and the feature vector $\left(V_{i}\right)$ for a new or unknown subject $i$.

The LZC [15][16][17][18][19] biomarker is used to assess the efficiency of the $\triangle \mathrm{EEG}_{\mathrm{A}}$ biomarker. In the LZC computation, the EEG signal is converted to a binary string as,

$$
x(i)=\left\{\begin{array}{lll}
0 & \text { if } & E E G(i)<M \\
1 & \text { if } & E E G(i) \geq M
\end{array}\right.
$$

where $x(i)$ is the equivalent binary value of $\operatorname{EEG}(i), i$ is the index of all values in the EEG signal, and $M$ is the median value of each EEG channel (for each EEG channel there is a median value). The median value is used to manage the outliers.

The binary string is then scanned from left to right till the end to produce new substrings. A complexity counter $c(N)$ is the number of the new substrings. The upper bound of $c(N)$ is used to normalise $c(N)$ to get an independent value from the sequence of length $N$. The upper bound of $c(N)$ is $N / \log _{2}(N)$. $c(N)$ is then normalised via $b(N)$ as,

$$
C(N)=\frac{c(N)}{b(N)}
$$

where $C(N)$ is the normalised value of the LZC, and $b(N)$ is the upper bound of the $c(N)$. The two reference feature vectors of the LZC contain the $C(N)$ for all EEG channels (one for normal, and the other for $\mathrm{AD}$ groups). As before, the Euclidean distance measure is used to discriminate between $\mathrm{AD}$ and normal subjects during classification.

\section{MATERIALS}

Two datasets (A and B) were obtained using a strict protocol from Derriford Hospital, Plymouth, U.K. and had been collected using normal hospital practices [11]. Dataset A consists of 3 Alzheimer's patients and 8 age-matched controls (over 65 years old) all of which have normal EEGs as confirmed by a consultant clinical neurophysiologist. Dataset B consists of 24 normal subjects and 17 probable $\mathrm{AD}$, which are not perfectly age matched. In the normal groups, the mean age is $69.4 \pm 11.5$ (minimum age is 40 and maximum age of 84 ), and $42 \%$ of the subjects are male. In the $\mathrm{AD}$ group, the mean age is $77.6 \pm 10.0$ (minimum is 50 and maximum is 93 ), and $53 \%$ of the subjects are male.

Dataset A was recorded using the traditional 10-20 system in a Common Reference Montage (by using the average of all channels as the reference) and converted to Common Average and Bipolar Montages in software. Dataset $\mathrm{B}$ was recorded using the modified Maudsley system that is similar to the traditional 10-20 system.

In both datasets, the EEG recordings include various states such as awake, hyperventilation, drowsy and alert, with periods of eyes closed and open. The sampling rate was reduced from $256 \mathrm{~Hz}$ to $128 \mathrm{~Hz}$ by averaging two consecutive samples for storage reasons. Fig. 1 shows the electrode locations in 10-20 system.

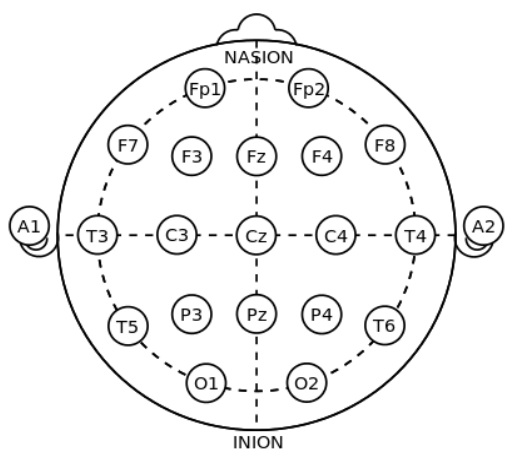

Figure 1. International 10-20 system.

\section{RESULTS AND DISCUSSIONS}

Following the approach in [11], complete recordings including artefacts were used without a priori selection of elements 'suitable' for analyses. This was to enable us to have an idea about the robustness and usefulness of the method in practice. Data from a fixed interval (61s to 240s) was used to avoid electrical artefacts, which regularly occur at the beginning of a record, therefore, give a standard three minute data to analyse.

The datasets were divided into AD and normal groups. In the development phase, 39 subjects from dataset B were used (24 normal, and 15 dementia) to create the two reference vectors - one for normal group and one for $\mathrm{AD}$ group. 
Dataset B was used to create the reference feature vectors because it is larger than dataset A. Consequently, it has more diversity and covered the most problem space.

In the testing phase, 13 subjects were used ( 2 dementia subjects from dataset A, 3 dementia subjects from dataset B, and 8 normal subjects from dataset $\mathrm{B}$ ) to create a feature vector for each subject.

The p-values using t-test was computed for mean $\triangle E E_{A}$ $\left(M_{c}\right)$ between $\mathrm{AD}$ and normal groups for each of the 21 electrodes to determine the most significant channels to be used to discriminate between AD and normal groups.

The Euclidean distances between the reference feature vectors and the feature vector of a new or unknown subject is then computed.

We classified a subject as a normal if their vector was closer to the reference vector of normal group than AD. Otherwise, we classified it as AD.

In this study, 6 channels of EEG (PZ, FZ, P4, CZ, F8, and T6) are used to detect $\mathrm{AD}$ by calculating the values of mean $\triangle \mathrm{EEG}_{\mathrm{A}}$ for each channel for each subject. These channels were selected based on an analysis of the mean $\Delta \mathrm{EEG}_{\mathrm{A}}\left(M_{c}\right)$ values for all channels for $\mathrm{AD}$ and normal subjects as shown in Fig. 3, and Table I. The results show that the $\triangle E E G_{A}$ values for $\mathrm{ADs}$ are lower than for controls. The reduction in $\triangle \mathrm{EEG}_{\mathrm{A}}$ values is thought to be due to the slowing in the EEG as a result of $\mathrm{AD}$ and this is in keeping with the finding in other studies [7][20].

TABLE I. MEAN $\triangle$ EEG $_{\mathrm{A}}$ FOR AD AND NORMAL GROUPS

\begin{tabular}{|l|l|c|c|}
\hline Seq. & Electrode & $\begin{array}{c}\text { Mean } \Delta \mathbf{E E G}_{\mathbf{A}} \text { for } \\
\text { AD group }\end{array}$ & $\begin{array}{c}\text { Mean } \Delta \mathbf{E E G}_{\mathbf{A}} \text { for } \\
\text { Normal group }\end{array}$ \\
\hline 1 & Fp1 & 32.423 & 39.458 \\
\hline 2 & Fp2 & 30.717 & 39.224 \\
\hline 3 & F7 & 30.500 & 35.485 \\
\hline 4 & F3 & 27.508 & 29.255 \\
\hline 5 & FZ & 12.847 & 27.550 \\
\hline 6 & F4 & 25.528 & 36.906 \\
\hline 7 & F8 & 29.014 & 42.259 \\
\hline 8 & A1 & 47.084 & 38.146 \\
\hline 9 & T3 & 41.691 & 36.914 \\
\hline 10 & C3 & 25.190 & 26.612 \\
\hline 11 & CZ & 12.910 & 24.082 \\
\hline 12 & C4 & 18.822 & 29.164 \\
\hline 13 & T4 & 29.282 & 40.599 \\
\hline 14 & A2 & 35.003 & 43.115 \\
\hline 15 & T5 & 39.464 & 45.539 \\
\hline 16 & P3 & 26.635 & 34.470 \\
\hline 17 & PZ & 20.170 & 33.909 \\
\hline 18 & P4 & 23.272 & 34.804 \\
\hline 19 & T6 & 34.020 & 45.597 \\
\hline 20 & O1 & 48.508 & 47.894 \\
\hline 21 & O2 & 41.767 & 43.528 \\
\hline & & & \\
\hline
\end{tabular}

Fig. 3 and Table II show that PZ channel (parietal lobe) has the minimum p-value, followed by FZ (frontal lobe), P4, CZ (central lobe), F8, and T6 (temporal lobe). This illustrates, the gradual slowing of brain wave activity due to
$\mathrm{AD}$, starts from the back of the brain (parietal lobe) towards the front (frontal lobe) and from right to the left side and this finding is consistent with the other studies [13][20][21][22][23]. In addition, occipital lobe is the last part of the brain that affected by AD.

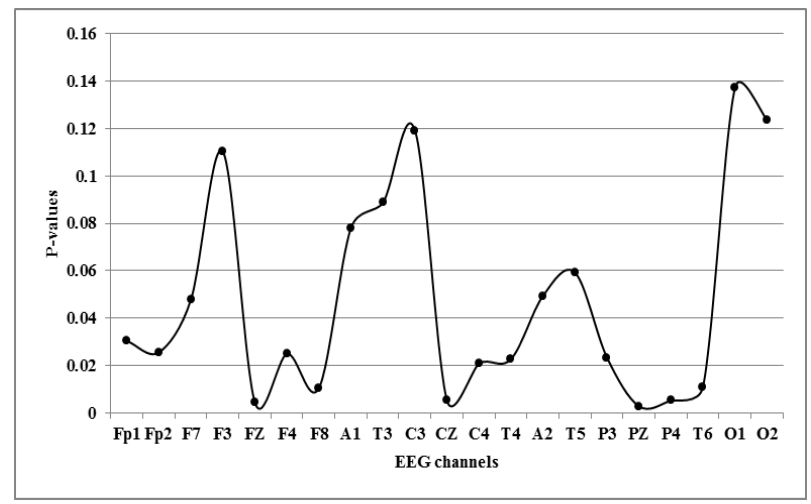

Figure 2. P-values between $\mathrm{AD}$ and normal groups.

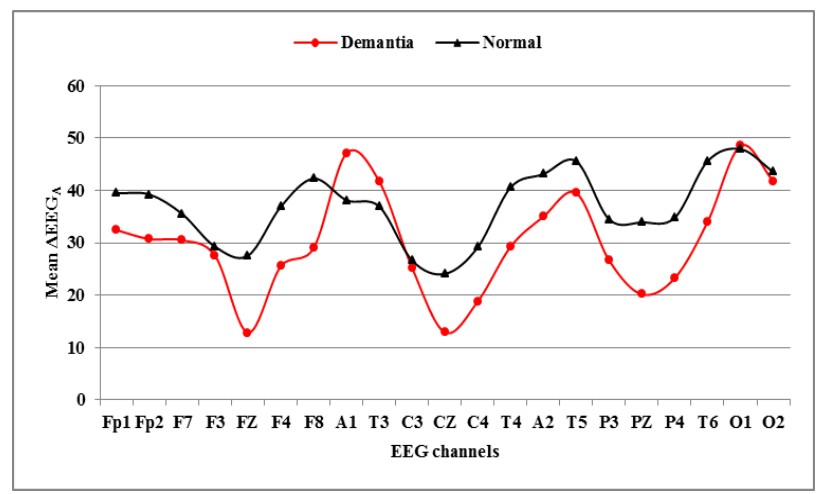

Figure 3. Demonstrates the mean $\triangle \mathrm{EEG}_{\mathrm{A}}$ for $\mathrm{AD}$ and normal groups.

TABLE II. P-VALUES BETWEEN AD AND HEALTHY GROUPS

\begin{tabular}{|l|l|c|}
\hline Seq. & Electrodes & P-values \\
\hline 1 & PZ & 0.0194 \\
\hline 2 & FZ & 0.0306 \\
\hline 3 & P4 & 0.0360 \\
\hline 4 & CZ & 0.0364 \\
\hline 5 & F8 & 0.0733 \\
\hline 6 & T6 & 0.0764 \\
\hline 7 & C4 & 0.1453 \\
\hline 8 & T4 & 0.1586 \\
\hline 9 & P3 & 0.1633 \\
\hline 10 & F4 & 0.1742 \\
\hline 11 & Fp2 & 0.1784 \\
\hline 12 & Fp1 & 0.2119 \\
\hline 13 & F7 & 0.3340 \\
\hline 14 & A2 & 0.3454 \\
\hline 15 & T5 & 0.4139 \\
\hline 16 & A1 & 0.5457 \\
\hline 17 & T3 & 0.6204 \\
\hline 18 & F3 & 0.7709 \\
\hline 19 & C3 & 0.8335 \\
\hline 20 & O2 & 0.8649 \\
\hline 21 & O1 & 0.9597 \\
\hline & & \\
\hline
\end{tabular}


The results of our study are consistent with other studies that found out that the slowing of the EEG is a marker for the subsequent rate of cognitive and functional decline in $\mathrm{AD}$ patients [12].

The performance of the $\triangle \mathrm{EEG}_{\mathrm{A}}$ biomarker was assessed by calculating its sensitivity, specificity, accuracy, precision and error rate. We compared the performance of the new approach with that of LZC approach. The results are summarised in Table III. It is seen that the new approach outperforms the LZC approach.

TABLE III. PERFormanCE RESUlts of $\triangle$ EEG $_{\mathrm{A}}$, AND LZC APPROACHES

\begin{tabular}{|l|l|l|}
\hline & DEEGA & LZC \\
\hline Sensitivity & $100.00 \%$ & $36.36 \%$ \\
\hline Specificity & $88.8888 \%$ & $50.00 \%$ \\
\hline Accuracy & $92.30 \%$ & $38.46 \%$ \\
\hline Precision & $80.00 \%$ & $80.00 \%$ \\
\hline Error rate & 0.0769 & 0.615 \\
\hline
\end{tabular}

\section{CONCLUSION}

Our results suggest that changes in EEG amplitudes, $\triangle \mathrm{EEG}_{\mathrm{A}}$ is a promising biomarker for AD. As AD subjects have significantly lower $\triangle \mathrm{EEG}_{\mathrm{A}}$ values., this provides an effective way to discriminate between $\mathrm{AD}$ patients and control subjects. Future work will evaluate the new approach using larger EEG datasets.

\section{ACKNOWLEDGMENT}

The first author would like to thank The Ministry of Higher Education and Scientific Research (MoHESR) - Iraq for their financial support. Financial support by the EPSRC is also gratefully acknowledged.

\section{REFERENCES}

[1] E. C. Ifeachor et al., "Biopattern analysis and subject-specific diagnosis and care of dementia," in Engineering in Medicine and Biology Society, IEEE-EMBS 2005. 27th Annual Int.l Conf. of the IEEE, pp. 2490-2493.

[2] M. Prince et al. "World Alzheimer Report 2015", August, 2015

[3] E. C. Ifeachor et al., "Nonlinear methods for biopattern analysis: role and challenges," in Proc. of the 26th Annu. Int. Conf. of the 2004 IEEE EMBS, pp. 5400-5406.

[4] A. L. Sutton, Alzheimer disease sourcebook. Detroit: Omnigraphics. Peter E. Ruffner Publisher, 2011, pp. 76.

[5] M. Weiner and Z. Khachaturian "The Use of MRI and PET for Clinical Diagnosis of Dementia and Investigation of Cognitive Impairment: A Consensus Report", Chicago, 2005.

[6] P. Zhao et al., "Characterization of EEGs in alzheimer's disease using information theoretic methods," in Engineering in Medicine and Biology Society, 2007 IEEE EMBS 2007. 29th Annu. Int. Conf. of the IEEE, pp. 5127-5131.

[7] A. H. Al-nuaimi et al., "Tsallis entropy as a biomarker for detection of Alzheimer's disease," in Engineering in Medicine and Biology Society, 37th Annual Int. Conf. of the IEEE, EMBC 2015, pp. 4166-4169.

[8] C. F. V. Latchoumane et al., "Dynamical Nonstationarity Analysis of Resting EEGs in Alzheimer's Disease," in Neural Information Processing Springer, vol. 4985, pp. 921-929, Jan. 2008.

[9] J. Dauwels et al., "On the early diagnosis of Alzheimer's disease from EEG signals: A mini-review," Advances in Cognitive Neurodynamics II Springer, pp. 709-716, 2011.
[10] C. Babiloni et al., "Sources of cortical rhythms change as a function of cognitive impairment in pathological aging: a multicenter study," Clin. Neurophysiol., vol. 117, no. 2, pp. 252-68, Feb. 2006.

[11] G. Henderson et al., "Development and assessment of methods for detecting dementia using the human electroencephalogram," IEEE Trans. Biomed. Eng., vol. 53, no. 8, pp. 1557-1568, Aug. 2006.

[12] J. Jeong, "EEG dynamics in patients with Alzheimer's disease," Clinical neurophysiology, vol. 115, no. 7, pp. 1490-1505, 2004.

[13] C. Babiloni et al., "Directionality of EEG synchronization in Alzheimer's disease subjects," Neurobiology of aging, vol. 30, no. 1, pp. 93-102, 2009.

[14] J. R. Evans and A. Abarbanel, eds. Introduction to quantitative EEG and neurofeedback. Elsevier, 1999, pp.56-57.

[15] D. Abásolo et al., "Lempel-Ziv complexity of cortical activity during sleep and waking in rats,". Journal of neurophysiology, vol. 113, no. 7, pp. 2742-52, Apr. 2015.

[16] A. Mateo et al., "Interpretation of the Lempel-Ziv complexity measure in the context of biomedical signal analysis," Biomedical Engineering IEEE Transactions, vol. 53, no. 11, pp. 2282-2288, 2006.

[17] D. Abásolo et al., "Analysis of EEG background activity in Alzheimer's disease patients with Lempel-Ziv complexity and central tendency measure," Medical engineering \& physics, vol. 28, no. 4, pp.315-22, May 2006.

[18] M. W. Rivolta et al., "Effects of the series length on Lempel-Ziv Complexity during sleep," in Engineering in Medicine and Biology Society, 36th Annual Int. Conf. of the IEEE EMBC 2014, pp. 693-696.

[19] A. Lempel and J. Ziv, "On the complexity of finite sequences. Information Theory," IEEE Transactions on Information Theory, Jan, vol. 22, no. 1, pp.75-81, 1976.

[20] B. Czigler et al., "Quantitative EEG in early Alzheimer's disease patients - power spectrum and complexity features," International Journal of Psychophysiology, vol. 68, no. 1, pp.75-80, 2008.

[21] C. Babiloni et al., "Fronto-parietal coupling of brain rhythms in mild cognitive impairment: a multicentric EEG study," Brain research bulletin, vol. 69, no. 1, pp. 63-73, 2006.

[22] U. A. Khan et al., "Molecular drivers and cortical spread of lateral entorhinal cortex dysfunction in preclinical Alzheimer's disease," Nature neuroscience. vol. 17, no. 2, pp. 304-11, Feb. 2014.

[23] C. Goh et al., "Comparison of fractal dimension algorithms for the computation of EEG biomarkers for dementia," in 2nd Int. Conf. on Computational Intelligence in Medicine and Healthcare CIMED2005, Jun. 2005, pp. 464-471. 